\title{
Percepción de la satisfacción sexual, los celos y la fidelidad en parejas practicantes del estilo de vida swinger
}

\author{
Eduardo Andrés Acuña Mora \\ Universidad de Iberoamérica.
}

\section{Resumen}

El estudio que aquí se presenta realiza una exploración de la percepción de la satisfacción sexual, la fidelidad y los celos en cinco parejas swinger. Dicha investigación fue realizada como proyecto de graduación para optar por el grado de Licenciatura en Psicología de la Universidad de Iberoamérica en agosto del 2019. En ella, se aborda la práctica swinger desde un acercamiento a diferentes postulados teóricos, mediante la realización de un análisis cualitativo de contenido con el cual se cruza la información brindada de los testimonios de las parejas entrevistadas, en relación con el material académico recopilado y estudiado previamente. Las conclusiones del estudio muestran que estas parejas swinger logran controlar sus sentimientos en relación a los celos, se perciben como fieles dentro del marco de los acuerdos que negociaron con sus parejas, y por último gozan de una plena satisfacción en el ámbito de su sexualidad.

Palabras clave: swinger, fidelidad, sastisfacción sexual, celos, percepción.

\begin{abstract}
This article constitutes an exploratory study of the perception about sexual satisfaction, fidelity, and jealousy in five swinger couples. This research project stems from a dissertation written in 2019 to obtain a Graduate Degree in Psychology in the Universidad de Iberoamérica. The article studies the swinger lifestyle as approached from different theoretical standpoints, through a qualitative Content Analysis that compares the information provided in the couples' testimonies with the academic material gathered beforehand. The conclusions of this study show that these swinger couples handle jealousy well, and perceive themselves as faithful within the frame of agreements that they have negotiated, as well as expressing full sexual satisfaction
\end{abstract}

Key Words: swinger, faithfulness, sexual satisfaction, jealousy. 


\section{Introducción}

La sexualidad humana constituye una de las áreas esenciales de la vida. Es considerada un derivado del instinto biológico asociado la reproducción, pero cuenta también con la participación del aspecto psicológico. Esta conjunción implica que la sexualidad humana sea compleja y amplia, pero rica en cuanto a la cantidad de matices y variantes según los cuales puede manifestarse y ejercerse.

Las sociedades humanas han experimentado grandes cambios a lo largo del tiempo; si bien muchos de estos se dieron a nivel económico y político, también los cambios en la cultura generaron un gran impacto en el estilo de vida de las personas. La globalización ha propiciado que en la actualidad muchas personas lleven un estilo de vida de pareja con una estructura distinta a la de sus padres y abuelos, quienes vivían en estructuras más rígidas que delimitaban su comportamiento con reglas sociales preestablecidas sobre lo que se esperaba de su comportamiento, su percepción y expresión del mundo. De esta manera, encontramos personas que, en la actualidad, han buscado diferentes dinámicas de vida que les permiten vivir con mayor libertad, de acuerdo con su sistema personal de valores que difiere del social seguido por sus antecesores.

Con el cambio de las dinámicas tradicionales surgieron modelos alternativos para vivir la relación de pareja, siendo estos la base de los matrimonios abiertos y las relaciones extraconyugales, lo cual sustenta la perspectiva de las parejas que han decidido llevar un modo de vida basado en la dinámica swinger.

Dichas prácticas se han ido extendiendo cada vez más en el mundo. A nivel internacional, se ha visto la evolución y crecimiento de los grupos practicantes del estilo de vida swinger, como se observa en los estudios académicos realizados en Estados Unidos por autores como Jenks (2014), Kimberly y Hans (2017), y a nivel de Latinoamérica, en países como Uruguay y Colombia, en los estudios de Monsalve et al (2014) y Olivera (2014). En el caso de Costa Rica, los mismos testimonios de las parejas entrevistadas así como las páginas web de contacto swinger ${ }^{1}$ señalan que este estilo de vida alternativo de parejas tiene muchos años de estar presente en el país, principalmente gracias a la existencia de espacios de reunión privados como clubes, bares, hoteles y áreas de reunión que se especializan en dar a esta población las condiciones necesarias para que realicen sus encuentros. Adicionalmente, el swinging es una temática que ya se ha tratado o mencionado en medios de prensa costarricenses; algunos ejemplos de estas publicaciones en prensa son: un artículo de prensa sobre el incendio del local del club swinger Pareja Total (Otey, 2018), un artículo de prensa sobre la confesión de un practicante del estilo de vida swinger sobre su vida en el dicho ambiente (Otey, 2018), un artículo de presa sobre un crucero para "parejas de mente abierta" (Otey, 2017) y un artículo de prensa sobre la experiencia que se vive en un club swinger

\footnotetext{
${ }^{1}$ No se incluyen los nombres y direcciones de estas plataformas web para proteger el anonimato de las personas que forman parte de esta comunidad.
} 
(Cruz, 2014). Sin embargo, en la búsqueda de antecedentes sobre la temática swinger a nivel nacional por medio de los repositorios universitarios OPAC, KIMUK, SIIDCA, KERWA y el portal de revistas académicas de la Universidad de Costa Rica, no se dispone aún de investigaciones de índole científica que puedan proporcionar información sobre estas poblaciones. El vacío existente en cuanto a estudios formales académicos en Costa Rica sobre este tema motiva la siguiente investigación, en el tanto la exploración de este tema permite esclarecer muchos de los mitos que existen alrededor de esta práctica, en especial en Latinoamérica, en donde el estilo de vida swinger ha sido poco estudiado.

Diferentes estudios señalan que dentro del proceso mismo de sus investigaciones han encontrado una resistencia a investigar la temática swinger en el ámbito académico. Distintos autores han considerado que quizás algunos investigadores ven el tema como un cuestionamiento a la moral, ya que, en muchos casos, al tratar este tema de investigación en el contexto de la universidad, se aludía con suspicacia a los motivos para elegir el objeto de investigación y al compromiso moral de los investigadores, juzgando de manera negativa a quienes deciden estudiar estas temáticas, señalándoles como personas inmorales por estudiar temas tabú (Olivera, 2014; Orejuela et al, 2012). Si bien el objeto central de dichos estudios ha sido la comprensión del estilo de vida swinger, existen interpretaciones variadas que responden al enfoque de las diferentes corrientes psicológicas mediante las cuales los resultados fueron contrastados.

Las investigaciones de Gómez y Martínez (2009), Gómez (2009), y Orejuela et al (2012) son muestra de la predominancia del enfoque psicoanalítico a la hora de abordar el tema. Los dos primeros estudios mencionan, según su enfoque, que el hombre podría acceder a que su pareja tuviera relaciones con otras personas como forma de compensar su incapacidad para satisfacerla en su rol de amante sexual. Sin embargo, Orejuela et al (2012) realizan una reflexión desde una perspectiva interdisciplinaria entre la sociología, la antropología y el psicoanálisis, mediante la que llegan a la conclusión de que la práctica swinger permite a las parejas el establecer un lazo afectivo diferente entre ellos; esto se logra mediante el cuestionamiento de la percepción tradicional de la moral y de la sexualidad, dando como resultado la creación de nuevas normas de interacción social que son antiestructurales a las establecidas por la sociedad. Por ende, los acuerdos con consentimiento de las partes llegan a convertirse en una nueva normativa para la interacción sexual y social con otros individuos.

Kimberly y Hans (2017), realizan estudios en distintas parejas que practican el estilo de vida swinger por medio de entrevistas y preguntas sobre su dinámica de pareja, la motivación, y los conceptos y reglas que tienen para la realización del acto sexual con otras parejas. Concluyen que aunque las parejas swinger son abiertas a la sexualidad con otros, mantienen reglas a nivel de pareja en cuanto a acuerdos para definir con quiénes comparten y con quiénes no. Entre sus reglas se encuentra el significado del "no" como respuesta no negociable si alguna de las partes así lo expresa, la no tolerancia a la agresión, el uso del condón, el lazo de confianza, la comunicación mutua y las propias reglas personales. 
En cuanto a la investigación de Monsalve et al (2014), estos realizaron un estudio sobre algunos aspectos del estilo de vida swinger, orientado a identificar cómo es la vivencia de las parejas con una relación estable que viven la actividad swinger. Indagaron sobre cuáles son los referentes que poseen de conceptos como amor, fidelidad y relación de pareja. Se utilizó el método de entrevista con las parejas, y se concluyó que, aunque ambos sujetos de la pareja entran en el estilo de vida swinger, existen algunas discrepancias en cuanto a las motivaciones para vivir dichas experiencias; sin embargo, se manejan percepciones similares en el tema del amor, y fidelidad.

La investigación de Hurtado y Serna (2011) consiste en un artículo que realiza un acercamiento a las precepciones sobre la fidelidad que se estructuran al interior de cuatro parejas swinger. En éste, se hace un análisis de las entrevistas acerca de su historia, su sexualidad y la regulación de las normas sociales. Posteriormente, se abordan las prácticas específicas y la forma percibida de la fidelidad.

Por su parte, Jenks (2014) realiza un estudio comparativo entre las parejas swinger y las poliamorosas, y aquellas que no caben dentro de ninguna de estas categorías. El estudio consistió en el análisis de una muestra de encuestas online completadas por 1400 individuos. Se compararon las percepciones en cuanto a afiliaciones políticas, religiosas, aceptación o rechazo de temas como el aborto, el matrimonio igualitario y la pena de muerte. Este estudio concluye que las personas poliamorosas tienden a ser democráticas y liberales, menos religiosas, apoyan más el aborto, el matrimonio igualitario, y la mayoría no acepta la pena de muerte. Por su parte, los swinger muestran no tener gran afiliación religiosa; la mayoría apoya el matrimonio igualitario, el divorcio, el aborto y no aceptan del todo la pena de muerte.

En cuanto al perfil de la persona que realiza esta actividad, Bergstrand y Williams (2000); Jenks (2014); Monsalveet al (2014); Kimberly y Hans (2017), llegan a observar en sus investigaciones que la práctica swinger existe en diferentes niveles socio económicos, políticos, religiosos y etarios, siendo la mayoría individuos de clase media alrededor de 40 años, de naturaleza política más liberal y de costumbres y prácticas religiosas menos conservadoras. Dichas investigaciones fueron realizadas en Estados Unidos por Bergstrand y Williams (2000), Jenks (2014), Kimberly y Hans (2017), y por Monsalve et al (2014) en Colombia. Además de observarse las normas creadas por cada pareja para la interacción con otros en sus encuentros sexuales y sociales, existe la presencia de un conjunto de normas establecidas por la comunidad swinger, el cual es el código base en el que esta comunidad se basa para sus normativas. Estas se caracterizan por el respeto, la tolerancia, la apertura de la orientación sexual (predominando una mayor aceptación de bisexualidad femenina sobre la masculina), las reglas de apertura y limitación de las distintas prácticas. Ejemplo de ello el uso obligatorio del preservativo y la no insistencia ante la presencia de un "no", llegándose a la conclusión de que la práctica swinger, además de ser una práctica sexual, llega a ser una práctica social, debido a que estas parejas se encuentran en un entorno de aceptación y respeto que no encuentran dentro de las interacciones sociales con aquellos fuera de estas prácticas (Kimberly y Hans, 2017). 
Las parejas practicantes del estilo de vida swinger muestran una preferencia por mantener su estilo de vida en secreto de las demás personas, en especial de familiares e individuos de poca confianza. De lo contrario, esto podría implicar un juicio social que puede generar como consecuencia un descrédito por parte de las personas que no aceptan aquellas prácticas que quedan por fuera del ideal heterocentrado (Olivera 2014). De igual manera, los participantes de este estudio expresaron que el swinging se considera una práctica tabú para la sociedad costarricense, lo cual podría deberse a una estructura moral conservadora basada en creencias religiosas que podrían contribuir a una percepción negativa de las prácticas sexuales alternativas.

En cuanto a los celos, éstos pueden generarse como un mecanismo de protección y supervivencia en situaciones en las que el individuo se ve amenazado o en peligro. Esta respuesta puede ser más intensa dependiendo del grado de importancia o vinculación que se tenga con quien se relaciona la naturaleza de esta amenaza, la cual puede ser real o imaginaria (Pines, 1992; Buss et al., 1999). Se observa que los motivos de los celos varían según el sexo, donde se ha observado que para los hombres una posible infidelidad sexual por parte de su pareja es una amenaza mayor debido a que podría afectar su descendencia y patrimonio, mientras que para la mujer una posible infidelidad afectiva por parte de su pareja podría representar una amenaza a su vínculo de pareja y la posible pérdida del cuido de sus hijos y la estabilidad para estos poder desarrollarse; todo esto está basado en el rol masculino de proveedor y protector, y el rol femenino de cuido y apego (Canto et al., 2001).

Para Morales (citado en Monsalve, 2009), el término pareja se define por la afiliación, la cual se entiende como la "la tendencia que presentan los seres humanos a buscar la compañía de ciertas personas desarrollando vínculos que generan seguridad y protección o que se toman como modelo de comportamiento" (p. 18). La afiliación con otras personas permite el desarrollo de la autoestima y la expresión de la sexualidad, ya que el poder compartir con otras personas permite vivir experiencias que serían difíciles de conseguir de manera solitaria.

Para Chaumier y Hernández (2006), existen modelos actuales de vida en pareja que son muy diferentes entre sí, y que han variado en sus formas y principios a partir de los sucesos contemporáneos de la sociedad, ya que las parejas actuales pueden vivir uno sin el otro sin conflictos y sin que falte el afecto. Las parejas actuales desarrollan sus propias reglas, las cuales les permiten una mejor convivencia mutua y una mayor apertura crítica con respecto a la conyugalidad. Como resultado de esto, se dan modelos distintos de la vida en pareja, entre los cuales se incluye el de la práctica swinger.

El concepto de infidelidad puede variar dependiendo de la percepción de la persona, y según lo que considere transgresor para sí misma. Por ejemplo, existen personas para las cuales, ante una infidelidad, la ausencia de coito entre los implicados hace que no se considere una infidelidad, o al menos no una grave. Para otras, los besos con una tercera persona son suficientes para considerar la interacción como una infidelidad. El factor determinante está en la ruptura del contrato acordado y negociado por la pareja sobre los 
límites y aperturas de la relación. Un ejemplo de esto es el acuerdo de las relaciones monógamas donde se acuerda exclusividad sexual y afectiva (Eisenberg, 1999).

En el caso de los swingers, los fundamentos del contrato de pareja suelen ser más liberales en comparación con aquellos de las parejas tradicionales, ya que llevan a cabo actividades sexuales con terceros. Sin embargo, las dinámicas de estas actividades varían según cada pareja. Por ejemplo, algunas realizan intercambios de pareja, otras pueden tener relaciones con un tercero únicamente si la pareja está presente, o bien pueden tener relaciones como quieran y con quien quieran (Camacho, 2004).

Las diferencias entre sexos reflejan una tendencia en las mujeres a percibir como más amenazante la infidelidad emocional de su pareja que la infidelidad sexual, mientras que en el caso de los hombres esta amenaza es más percibida ante una infidelidad sexual que una emocional (Canto et al., 2001; Buss et al., 1999).

La fidelidad en la cultura swinger se interpreta como la ausencia del engaño a la hora de realizar el acto sexual, al haber negociado y aprobado el individuo los términos con su pareja sobre cómo se darían estos actos; en este sentido, se definen tantos los aspectos sexuales como los emocionales (Vanegas, 2011).

Las relaciones no monógamas consensuadas (NMC) engloban un conjunto de relaciones en las que los individuos están de acuerdo en vivir múltiples experiencias sexuales, románticas y/o emocionales con otras personas (Barker y Langdridge, 2010; Conley et al., 2012; Conley et al., 2013). Dicho de otra forma, estas relaciones buscan de manera intencional la no-monogamia (Noël, 2006), la no-monogamia negociada y sin secretos (Jamieson, 2004), o una no-monogamia responsable (Anapol, 1997; Klesse, 2006; Lano y Parry, 1995). Estas relaciones, por medio de acuerdos negociados entre los dos miembros de la pareja, establecen qué aperturas y limitantes a nivel sexual y emocional tendrá la relación.

En el caso de los swinger, la principal característica que poseen como un tipo de relación NMC es el relacionarse sexualmente con otras personas en un entorno social como lo serían las fiestas o convenciones swinger (Jenks y Smith, citados en Mastick et al., 2014)

Según Mastick et al (2014), las relaciones NMC se pueden describir principalmente en tres categorías con sus particularidades: relaciones swinger, relaciones poliamorosas y relaciones abiertas; dichas serán escritas a continuación.

Las relaciones swinger se definen como parejas que se encuentran en el ambiente swinger, y en las cuales ambos miembros deciden tener relaciones sexuales con otras personas más allá de su pareja. Los vínculos para estas relaciones suelen conseguirse en fiestas o algún otro entorno social. Las personas que realizan estas actividades tienen claro que no son monógamos sexualmente, pero sí emocionalmente, por lo que después de involucrarse con terceros la relación vuelve a ser el centro de la pareja.

Las relaciones poliamorosas se definen como aquellas parejas que deciden tener relaciones emocionales, románticas y sexuales con más de una persona. Las personas involucradas en estos vínculos tienen conciencia de que no son monógamos. Esta nomonogamia es decidida de manera consensual entre todos los involucrados en las relaciones. 
Sin embargo, hay algunas personas poliamorosas que buscan ampliar los vínculos emocionales y románticos, pero sin tener relaciones sexuales con las otras personas.

Las relaciones abiertas están constituidas por aquellas parejas que desean experimentar relaciones sexuales con otras personas además de su pareja. Estas parejas tienen claro que no son monógamos. Esta no-monogamia es decidida de manera consensual por la pareja. Los encuentros sexuales suelen suceder de manera individual, es decir, sin la pareja presente, por lo que estos encuentro se dan de manera independiente.

Existe una confusión en cuanto a la definición de lo que significa ser swinger, ya que las personas suelen confundir o mezclar la forma en que los swinger y las parejas poliamorosas llevan sus respectivas relaciones. En tanto los swinger se definen como aquellas personas que tienen intercambios de pareja con el propósito de vivir experiencias sexuales sin involucrar el aspecto emocional de pareja, llevando este estilo de vida en oposición a la estructura normativa de la sociedad, las personas poliamorosas se definen como aquellas personas que tienen relaciones románticas con otros individuos además de su pareja, creando vínculos afectivos en los que terminan involucradas tres o más personas, bajo los preceptos de la honestidad, la integridad y el respeto (Jenks, 2014).

La presente investigación se enfoca en estudiar el estilo de vida de las parejas que realizan prácticas swinger, así como su percepción de los celos, la satisfacción sexual y la fidelidad.

\section{Método}

El método de investigación utilizado para el diseño y análisis de resultados de esta investigación fue el análisis cualitativo de contenido estructurante (Flick et al., 2004), el cual tiene como objetivo filtrar aspectos específicos del material para realizar un análisis cruzado que permita una serie de criterios definidos por categorías preestablecidas, mediante procedimientos formales que van a depender de las dimensiones estructurantes definidas por la teoría. Por ello, esta investigación cruza la información recolectada de las entrevistas de los participantes con la de la teoría utilizada, para crear los criterios de las categorías por investigar.

\section{Participantes}

Se contó con la participación de 5 parejas entre 30 y 54 años, resultando en 4 personas en sus treinta, 4 en sus cuarenta y 2 en sus cincuenta. Cuatro de las parejas entrevistadas están casadas y una pareja vive en unión libre. Tanto los casados como aquellos en unión libre cuentan con un tiempo de convivencia de entre 10 y 20 años, siendo que 4 de las parejas superan los 10 años de convivencia. Las parejas entrevistadas tienen entre 2 y 18 años de practicar el estilo de vida swinger. En cuanto a sus creencias religiosas, 8 de los entrevistados se definen como católicos, mientras que 2 se definen como ateos. En cuanto a su nivel educativo, 4 de los participantes terminaron la secundaria, 3 el bachillerato universitario, 2 
la licenciatura y 1 el doctorado. En cuanto a la orientación sexual, 5 de los entrevistados se define como bicuriosos/as, 1 como bisexual y 4 como heterosexuales.

\section{Instrumentos}

El instrumento utilizado para este estudio fue la entrevista semiestructurada. Para Martinez (1998), este tipo de entrevista permite generar una guía de entrevista con preguntas agrupadas por temas o categorías, con base en los objetivos del estudio y la literatura sobre el tema. Tiene un formato flexible que permite al entrevistador tener mejor interacción con el entrevistado, previniendo que este último experimente desaprobación respecto a sus testimonios, y permitiendo al entrevistador mostrarse más receptivo y sensible a lo que el entrevistado exprese. Las preguntas deben poseer un formato que permita al entrevistado hablar de manera más libre y espontánea.

Por consiguiente, para esta investigación se generó una serie de preguntas de tipo abierto con el objetivo de explorar la percepción de los celos, la satisfacción sexual, la fidelidad y las experiencias vividas por las parejas entrevistadas. Las preguntas están divididas en 5 categorías por estudiar, que son la experiencia de la vivencia swinger, la percepción de la satisfacción sexual, la percepción de la fidelidad, la percepción de los celos, y la vivencia de la sociedad y la familia.

Se empleó también un cuestionario digital que consiste en un formulario desarrollado en la plataforma de Google Forms. Se escogió esta plataforma por su facilidad para poder generar el cuestionario en formato online y que este pueda ser abierto en cualquier dispositivo o computadora, además de que esta plataforma recopila y ordena los datos del cuestionario por categorías. Este cuestionario consiste en preguntas cerradas orientadas a obtener información sociodemográfica y criterios individuales sobre la experiencia de la vivencia swinger, la percepción de la satisfacción sexual, la percepción de la fidelidad, la percepción de los celos, la sociedad y la familia.

\section{Procedimientos de recolección}

En primer lugar, se contactó a las parejas participantes por medio de páginas web especializadas para swingers, por perfiles de Facebook utilizados por parejas swinger para mantener contacto con otras parejas de la comunidad swinger, y por medio de referencias personales dadas por las parejas mismas sobre otras parejas swinger interesadas. Luego se concertó una cita con las parejas en un punto de encuentro y hora negociado entre las parejas y el investigador. Cada pareja se citó en días diferentes. Ya en el sitio establecido por cada pareja en las citas acordadas ${ }^{2}$, se le explicó a la pareja en qué consistía el estudio, los lineamientos que lo rigen, y se procedió a explicar el consentimiento informado, para que luego las parejas lo firmaran. Después de eso, se procedió a aplicar los instrumentos de investigación que consistieron, en primer lugar, en la aplicación individual del cuestionario a ambos miembros de la pareja, facilitándoles para este efecto una tablet para así garantizar

\footnotetext{
${ }^{2}$ Algunos preferían sitios públicos como cafeterías o restaurantes en donde se sentían cómodos para hablar del tema
} 
que la información brindada por cada uno fuera personal y no estuviera sesgada por la presencia de su pareja. En segundo lugar, se procedió a realizar la entrevista a la pareja en conjunto, utilizando las preguntas de la entrevista semiestructurada diseñada para este fin, procediendo el investigador a tomar nota de las respuestas respectivas. Posteriormente, se realizó un análisis cualitativo de la información obtenida tanto en la entrevista como en el cuestionario, para así comparar las respuestas de las parejas, buscar puntos en común, discrepancias, y así crear una definición de los puntos explorados en esta investigación. Finalmente se realizó el análisis de los datos con la base teórica y lo aportado por las parejas, para crear así un consenso en cuanto a la vivencia de las parejas swinger. El tiempo aproximado de cada entrevista fue de aproximadamente 1 a 2 horas.

\section{Procedimientos para mantener la confidencialidad y protección de los sujetos}

Se proporcionó a los participantes un consentimiento informado, el cual consiste en un documento que explica los derechos, reglas y beneficios de participar en el estudio en cuestión, y deja por sentado que el participante accede a formar parte del estudio. Este documento se les debe leer a los participantes antes de que éstos lo firmen, y se les debe entregar una copia.

Se utilizaron seudónimos en el estudio para proteger la identidad de los participantes. La identidad correspondiente a cada seudónimo es únicamente conocida por parte del investigador. De esta manera, se utilizarán únicamente los seudónimos para identificar la información correspondiente a cada participante.

\section{Procedimiento de análisis}

En cuanto al análisis de los datos, se utilizó el análisis cualitativo de contenido estructurante. Como primer paso para la realización del análisis, se desarrolló un estudio teórico de las categorías previamente seleccionadas para el estudio. Estas categorías fueron definidas con base en los temas teóricos presentes en las investigaciones existentes sobre la temática swinger. Siendo estas categorías similares a las de otros estudios como los de Monsalve et al (2014), para este estudio se optó por analizar la percepción de la satisfacción sexual, los celos, la fidelidad en parejas que ejercen el estilo de vida swinger.

Posteriormente se procedió a sistematizar la información de los participantes del estudio. Para esto, se tomaron los fragmentos de lo anotado durante las entrevistas semiestructuradas y la información contenida en los cuestionarios. A cada pareja se le asignaron pseudónimos para proteger su identidad y se analizaron los aportes a la luz de las categorías diseñadas para la investigación. Por lo tanto, cada pareja aportó información sobre cada una de las categorías.

Luego se realizó un análisis cruzado que compara las definiciones de la teoría con lo expresado por las parejas, para así determinar las similitudes y diferencias existentes. Al haber similitudes entre la teoría y las entrevistas, este procedimiento permite explorar y analizar cómo estas parejas viven sus experiencias como parejas swinger. 
Finalmente, para lograr determinar las similitudes y diferencias del análisis cruzado de los datos, se buscó la presencia de una serie de subcategorías previamente establecidas en el contenido de los testimonios obtenidos mediante entrevista, intentando determinar una valoración de cada subcategoría por parte de los sujetos. A partir de esto, se llega a establecer una confirmación de los postulados planteados por los estudios previos en relación con estas categorías prestablecidas.

\section{Resultados}

Para el análisis de los resultados, se revisó el análisis cruzado de la información de la teoría en relación con aquella obtenida de los testimonios en las entrevistas. Se analizó la percepción de estas parejas en cuando a su satisfacción sexual, la percepción de la fidelidad y la percepción de los celos. Al cruzar los testimonios de la experiencia subjetiva de los participantes, pero estructurada por medio de los instrumentos de investigación, se pudo observar similitudes con la teoría utilizada para determinar las categorías de análisis preestablecidas para la investigación.

En cuanto al proceso de ingreso al ambiente swinger, se puede observar en estas parejas una búsqueda de alternativas para mejorar la relación de pareja. Algunas de las parejas que buscan experimentar la dinámica swinger lo hacen por exploración y búsqueda de nuevas experiencias, otras para generar unión en la pareja y salir de la rutina y la monotonía, y otros buscan esta alternativa para rescatar su relación de una posible separación, pero generando una apertura como solución en vez de separarse bajo el pensamiento tradicional de traición o infidelidad. Ejemplo de esto se ve en lo dicho por la pareja número 1:

"Comenzamos en el ambiente debido a una experiencia espontánea, en la cual con una amiga de confianza tuvimos un trío. Luego de eso, tuvimos mucha curiosidad por ver qué más se podía experimentar y usábamos páginas para poder conocer personas. Fue así como conocimos un grupo de swingers que hacían reuniones para conocerse y socializar. Así fue como conocimos el ambiente $y$, poco a poco, fuimos experimentando nuevas experiencias. A ambos esto nos llena, pues sentimos que cada cosa que hacíamos más nos unía y más nos gustaba vivir nuestras fantasías."

Por su parte, la pareja número 3 menciona:

"Nosotros iniciamos en nudismo. Yo practiqué nudismo más joven y, después de casados, decidí incluirla en esto. Teníamos 4 años de casados para ese momento. Un día le dije que fuéramos a una playa nudista y le gustó. Decidimos ir probando cosas para salir de la rutina, curiosear, disfrutar nuevos placeres, el morbo de ver tu pareja con otra persona. No lo hablamos mucho, 
sino que fuimos viviendo el momento. Conocimos una pareja que tenían unos 60 años, y fueron quienes más consejos y lecciones nos dieron para experimentar en este ambiente."

Varias de las parejas entrevistadas narran sus inicios en el ambiente swinger, los pasos que dieron, y la perspectiva que desarrollaron para acomodar sus reglas, acuerdos y condiciones para poder ejercer con libertad este estilo de vida. Estos acuerdos se ven reflejados a continuación en el testimonio de cada una de las parejas, en cuanto a cuáles son las reglas que ellos definen:

Pareja número 1: "No es no; no emociones; es un juego, no se involucra lo emocional; proteger la identidad de las personas; uso del condón."

Pareja número 2: "No es no; se debe tener buena higiene personal; no mezclar emociones; no revelar a las personas que se es swinger; uso del condón."

Pareja número 3: "No es no; tener buena higiene personal; verse arreglado; oler bien; no tener un vocabulario vulgar; ser respetuosos; uso del condón."

Pareja número 4: "No es no; no emocional; respeto de los límites; uso del condón." condón.”.

Pareja número 5:"No es no; respetar lo que se quiera hacer; uso del

Éstas muestran ser relaciones con mucha comunicación y gran énfasis en la transparencia; invitan a la pareja al diálogo sin tabús, explorando opciones y negociando límites y experiencias por vivir, siempre dentro del marco de la complicidad, el respeto y la lealtad.

Aunque parte del desarrollo de estas parejas se basa en alcanzar la libertad para vivir sus experiencias sexuales sin miedos y con transparencia, logran esto por medio de mecanismos que deben estar presentes en todo modelo y tipo de pareja, ya que generan mecánicas de comunicación, lealtad, complicidad, y negociación de acuerdos y reglas formadas bajo el criterio de ambas partes y que se sustentan en el respeto al otro. Por ejemplo, en los fragmentos mencionados anteriormente se puede observar cómo las 5 parejas tienen en común, como reglas básicas y fundamentales, el respeto al "no" y el uso de preservativo en todo momento. Tomando en cuenta lo dicho por Acuña (citado en Monsalve, 2009), la relación de pareja está conformada por dos personas que han tenido, tienen y tendrán un continuo en sus expresiones de amistad, comunicación intimidad, sexo y pasión. Estos 
individuos dejan en claro que la persona con quien comparten su vínculo emocional y su plan de vida es su pareja, siendo ésta el centro de lo que se comparta juntos. Las actividades swinger, entonces, son ejecutadas como un disfrute físico en relación con lo sexual, y como una interacción social con quienes comparten estas dinámicas. Se cumple así lo expresado por Morales (citado en Monsalve, 2009), quien define que, en la búsqueda de una relación equilibrada, se espera una consistencia cognitiva, por lo que el individuo buscará alguien que tenga las mismas aficiones, ideas y gustos, para de tal manera sentir atracción hacia esa persona.

En cuanto al concepto de fidelidad de estas parejas swinger, este se basa en un respeto de los acuerdos y las reglas negociadas y establecidas bajo el consentimiento y opinión de ambas partes de la pareja. El seguimiento de estos acuerdos genera vínculos de confianza y lealtad, que son valores buscados en una relación, como lo dicho por la pareja número 2 :

"La fidelidad es un 50/50, que si él quiere algo y me lo dice y si yo estoy de acuerdo se hace. Fidelidad es que él me lo comente y no que haga a escondidas; es que si él va con otra mujer me lo va a decir [...] Infidelidad para nosotros sería el rompimiento de nuestros acuerdos, lo que hemos negociado y pactado, ya que nuestra idea es siempre tener la confianza de hablar lo que sea, por lo que no tener comunicación y ocultar cosas y romper acuerdos es nuestra definición de infidelidad".

En este testimonio podemos observar un ejemplo de lo que menciona Martín (2004): en el caso de los swingers, los fundamentos de su contrato con la pareja suelen ser más liberales en comparación con las parejas tradicionales, ya que realizan actividades sexuales con otras personas. Sin embargo, las dinámicas involucradas varían según cada pareja. Por ejemplo, hay parejas que realizan intercambios, pueden tener relaciones con un tercero sólo si la pareja está presente, o pueden tener relaciones como quieran y con quien quieran.

La fidelidad, como se conoce de manera tradicional, es cambiada por la negociación y la generación de acuerdos, donde ambas partes rompen tabús en pro de vivir experiencias nuevas, en un contexto más libre y bajo términos con los que se sientan bien. Con base en esto, las parejas swinger son fieles, ya que buscan el equilibro, la negociación, y la mejora de la comunicación con la pareja, todos elementos necesarios para una relación de pareja estable. Esto se da en especial cuando dejan en claro que esta apertura negociada es en términos sexuales y no emocionales, buscando que el centro siempre sea la pareja y la relación. Ejemplo de esto se ve en lo mencionado por la pareja número 5:

"Una regla importante que tenemos es que no hay involucramiento emocional de ninguno de los dos con las demás personas, para dejar claro nuestro lugar como pareja..." 
En este aspecto, las parejas swinger no son distintas a las parejas normativas. La diferencia radica en que la fidelidad es un concepto negociado y no uno asumido o impuesto por la sociedad. Otro ejemplo proporcionado por la pareja número 5 es el siguiente:

"Existen reglas preestablecidas, que han sido negociadas con base a lo que queremos disfrutar y lo que no. Ejemplo es como estar en el mismo espacio físico con la pareja con la que hacemos intercambio; no sentirse desatendidos por el otro. Hay reglas que fueron cambiando con el tiempo. Al inicio a mi pareja no le gustaba que yo besara a la otra chica, pero después esa regla cambió y ahora no es un problema."

Por otra parte, la infidelidad para estas parejas consiste en el rompimiento de estos acuerdos, asumiéndose éste como un ataque a la relación y al vínculo afectivo. Esto concuerda con lo dicho por Eisenberg (1999), quien define la infidelidad como la ruptura de un contrato, acuerdo o pacto implícito o explicito, cuando uno o ambos miembros de la pareja tienen algún tipo de relación con una tercera persona. Sin embargo, en concreto, se considera infidelidad la ruptura del contrato o acuerdo que se tiene dentro de la relación.

En cuanto a la percepción de los celos, estas parejas los definen como un sentimiento que se da cuando no existe una buena comunicación, confianza, seguridad y satisfacción con la pareja. Como ejemplo tenemos lo mencionado por la pareja número 4:

"Siempre hemos sido muy tranquilos en el tema de los celos. Nos sentimos muy seguros, nos damos mucha confianza. Para nosotros los celos son inseguridad y desconfianza de tu pareja... No sentimos que los celos nos afecten, pues confiamos plenamente en el otro. Basamos nuestra relación en comunicación y confianza. Disfrutamos con gusto las cosas sabiendo que el otro estará ahí y respetando lo acordado; entonces no nos sentimos amenazados. Sentimos que hay mucha seguridad en el otro, y sentimos que esta seguridad y confianza ha aumentado aún más después de entrar en el ambiente swinger, ya que podemos expresarnos más y sin miedos. Sentimos un mayor acercamiento y una mayor comunicación."

Las parejas entrevistadas expresan no sentir estos celos, ni sentirse amenazados en algún aspecto de la relación, debido al nivel de comunicación y confianza. Basan su relación en la transparencia y, al tener una dinámica sexual abierta a otras personas, no caen en los mismos roles de inseguridad de las parejas normativas. Incluso si se sienten afectados de alguna manera, abren la puerta al diálogo. 
Lo anterior concuerda con lo establecido por Pines (1992) y por Buss et al., (1999), quienes describen los celos como una emoción compleja que surge de las sospechas reales o imaginarias de una amenaza a la relación y al vínculo que es valioso para la persona. Por ende, los celos funcionan como un mecanismo de advertencia cuando la relación se encuentra en una posible problemática debido a temas como la infidelidad, lo cual, en muchos casos, es causa de divorcio y maltrato conyugal. Es posible que, al poseer las parejas swinger buenas mecánicas de comunicación, eviten de esta forma, la existencia de dudas o inseguridades, formando así un lazo más sólido.

Los celos, como emoción compleja que son, están influidos por múltiples variables, entre las que se pueden destacar factores culturales y características psicológicas de la persona que siente celos, así como aquellas otras variables que son propias de la situación y del tipo de relación, coincidiendo con lo dicho por White y Mullen (citados en Canto et al., 2009).

Las estrategias de comunicación y apertura que estas parejas desarrollan son lo que podría permitirles el desarrollo de una confianza exitosa con la pareja. Lo que les permite a los swinger llevar el estilo de vida que tienen es el rompimiento de los tabúes sexuales, convirtiendo así el sexo con otros en un tema de complicidad y no en un motivo de conflicto, como sucede en algunas ocasiones en las relaciones más normativas.

En cuanto a la percepción de la satisfacción sexual, las parejas estudiadas afirman sentirse satisfechas con el nivel de placer que experimentan en sus actividades swinger y en su interacción de pareja. Describen un incremento del placer y la excitación cuando se está interactuando sexualmente con otros. Un ejemplo de esto lo menciona la pareja número 1:

"Solemos tener sexo entre nosotros cada vez que podamos en la semana, por lo que solemos al menos hacerlo 2 o más veces. En el ambiente swinger solemos tener meses más activos que otros, pero en los más activos tenemos unos 4 encuentros en el mes. Estos encuentros pueden ser tríos con hombres, cuckold, intercambio, o hacerlo junto a otras parejas en las fiestas swinger. Ambos sentimos que experimentar con otras personas nos aumenta mucho el deseo sexual y la satisfacción que sentimos es muy sabrosa."

Este sentimiento parte de la complicidad que las parejas expresan sentir a la hora de vivir estas experiencias. Adicionalmente, expresan un aumento o mantenimiento del nivel de libido sexual cuando interactúan sexualmente como pareja, reforzándose así el vínculo de la relación. 
En cuanto a la percepción social, lo referido por las parejas entrevistadas apunta a que los practicantes del estilo de vida swinger son percibidos de manera negativa por la sociedad normativa. A pesar de que sus relaciones se pueden considerar como una forma alternativa de la vivencia de la sexualidad y una alternativa del modelo de pareja, se les señala como promiscuos e inestables, creándose así una imagen negativa que no les permite expresar con libertad su forma de pensar y vivir este estilo de vida. Ejemplo de esto lo menciona la pareja número 1:

"Sentimos que la sociedad discrimina mucho a los swinger. En algunas ocasiones he callado cuando oigo que se habla mal del ambiente o de la percepción que tienen de nosotros y en otras he defendido el tema. Sentimos que esto es algo normal y que no debería ser mal visto. Nos critican principalmente desde el punto de vista religioso y la moral social. Hasta el momento, ningún familiar de nosotros sabe que somos swinger y unos pocos amigos cercanos y de confianza sí lo saben... Mantenemos esto en privado por evitar problemas a futuro en los trabajos o la crítica que las personas nos puedan hacer."

Por este motivo, estas parejas viven su estilo de vida de manera hermética y sólo pocas personas cercanas llegan a saber sobre su participación en el ambiente. En cuanto a esta apertura, son los familiares las personas que estas parejas menos desean que sepan sobre su vida privada, por temor a ser víctimas de críticas y señalamientos. Ejemplo de esto se ve en lo mencionado por la pareja número 2:

"Sólo una persona de mi familia sabe que somos swinger y algunos amigos de confianza... Sentimos que estar en el ambiente swinger es algo bueno, a pesar de que para muchas personas es algo que se percibe negativo... Nadie de nuestros amigos ofamiliares sabe que somos swinger; es algo que dejamos para nosotros. Sentimos que quienes no saben cómo es el ambiente critican sin saber, ya que mucha gente nos trata de enfermos sexuales, que es una 'voladera parejo', y no saben del ambiente y sus reglas, ni de que es algo más social que sexual. $Y$ se siente extraño cuando oyes gente hablar mal de los swinger y no poder decir nada para defender el tema para mantener la discreción."

En cuanto a las experiencias sexuales vividas por estas parejas se observa que a las 5 parejas les gustan las dinámicas de tríos con hombres ${ }^{3}$, la dinámica cuckold $^{4}$ y los intercambios de pareja, como lo mencionado por la pareja número 2 :

\footnotetext{
${ }^{3}$ Son los tríos en los que la pareja invita a otro hombre para interactuar con la mujer; en esta dinámica el esposo y el invitado interaccionan con la mujer.

${ }^{4}$ En esta dinámica el invitado tiene sexo con la mujer, pero el esposo sólo observa sin participar del acto de manera directa.
} 
Psicolnnova, 5(1), 2021, 27-45

"Después de cada encuentro swinger sentimos muchas ganas de estar juntos. Sentimos que nuestra libido aumenta mucho y que deseamos hacer más juntos. Esa es la complicidad que nos tenemos: nos saca de la rutina, me hace descubrir cosas sobre mi cuerpo que antes no conocía, como mi capacidad de hacer squirt [eyaculación femenina]. Antes tenía que auto-estimularme para lubricar, ahora antes del acto sexual lubrico, y después lubrico más y más... Yo, al ver que ella está tan mojada, yo de solo verla se me erecta el pene más rápido.”

Esta misma pareja practica la dinámica de gangbang ${ }^{5}$ la cual expresan disfrutar como dinámica sexual y como refuerzo del vínculo de pareja:

"Él: Solemos tener sexo entre nosotros unas 4 a 6 veces al mes, y en el ambiente swinger 1 o 2 veces. Por lo general hacemos tríos con chicos, gangbang de hasta con 7 chicos hemos hecho, y yo disfruto la dinámica de cuckold. Disfruto verla a ella disfrutar, sólo observar o participar; en las dos me siento muy excitado y emocionado... Ella: La libertad que Arty me hace sentir es grande. Sé que puedo dejarme llevar y concentrarme en el disfrute, y sé que él está disfrutando y a la vez está pendiente de mí y cuida que esté bien... Vela por que los chicos me respeten, incluso cuando ya estemos teniendo sexo. Es una complicidad la que sentimos, que nos une y que sabemos que ambos hemos llegado a este punto en nuestra relación..."

El intercambio de pareja es una dinámica que las 5 parejas mencionan disfrutar y que les ha generado más excitación y complicidad cuando viven sus encuentros, como lo mencionado por la pareja número 4:

"Ella: Nosotros solemos tener sexo unas 3 veces al mes, y sí hacemos algo de ambiente de 1 a 2 veces en el mes, aunque a veces hacemos reuniones como solo para socializar con las parejas. Siento que mi deseo sexual aumenta mucho después de realizar una actividad como un intercambio, y después de eso deseo más estar con Tommie sexual y emocionalmente... Él: Mi deseo sexual es el mismo, no siento que aumente o disminuya en sí. Disfruto mucho la actividad, pero no siento un aumento de mi deseo sexual, sino que es el mismo con el que empecé todo. Aunque cuando la veo a ella con el otro chico, sí siento más euforia y más energía; me gusta verla disfrutar... Ella: Disfrutamos mucho el momento; me enfoco mucho en lo que hago con el chico que esté compartiendo en ese momento y, a la vez, me gusta verlo a él disfrutar con la otra chica."

\footnotetext{
${ }^{5}$ El gangbang es una dinámica sexual en la que tres o más hombres tienen sexo con una mujer en la misma habitación y momento.
} 


\section{Conclusiones}

La investigación llegó a encontrar las similitudes de la teoría con lo indicado por las parejas. Se observa una vivencia de la sexualidad libre y una dinámica basada en la negociación de acuerdos que permiten generar lazos de confianza y complicidad. Esto les permite vivir dinámicas sexuales variadas, desde tríos, intercambios de pareja y hasta dinámicas grupales como los gangbang. Reconocen la existencia de los celos, pero los manejan por medio de los acuerdos y la comunicación. Perciben la fidelidad como el respeto y seguimiento de los acuerdos donde se genera un vínculo de confianza.

En cuanto a las limitaciones del estudio, estuvieron principalmente en el tiempo para el desarrollo y recolección de datos y contacto de los participantes. Es importante, de realizarse un estudio posterior, tomar en cuenta que los swinger son herméticos al tratar de no revelar su estilo de vida, por lo que esto puede dificultar el acceso a sujetos de estudio. Otra recomendación sería crear posteriormente un diseño de investigación que permita hacer la entrevista semiestructurada no sólo con la pareja en conjunto, sino también de manera individual con cada miembro de la pareja, para así poder obtener información aún más detallada.

\section{Referencias}

Anapol, D. (1997). Polyamory the new love without limits: Secrets of sustainable intimate relationships. IntiNet Resource Center.

Barker, M., y Langdridge, D. (2010). Whatever happened to non-monogamies? Critical reflections on recent research and theory. Sexualities, 13(6), 748-772. https://doi.org/10.1177/1363460710384645

Bergstrand, C. y Williams, J. (2000). Today's Alternative Marriage Styles: the Case of Swingers. Electronic Journal of Human Sexuality, 3, 1-9. http://www.ejhs.org/volume3/swing/body.htm.

Buss, D., Shackelford, T., Kirkpatrick, L., Choe, J., Lim, H., Hasegawa, M., . . . Bennet, K. (1999). Jealousy and the Nature of Beliefs about Infidelity: Test of Competing Hypothesis about Sex Differences in the United States, Korea and Japan. Personal Relationships, 6, 125-150.

Camacho, J. (2004). Fidelidad e infidelidad en las relaciones de pareja. Editorial Dunken. doi:9789870205883

Canto, J., García, P. y Gómez, L. (2001). Reacción de celos ante una infidelidad. Diferencias entre hombres y mujeres y características del rival. Psicothema, 13(4), 611-616. http://www.redalyc.org/pdf/727/72713413.pdf

Canto, J., García, P. y Gómez, L. (2009). Jealousy and Emotion: How Partners React to Infidelity. Athenea Digital, 15, 39-55. doi:10.5565/rev/athenea.528.

Chaumier, S. y Hernández, M. (2006). El nuevo arte de amar. Alianza Editorial. 
Conley, T. D., Ziegler, A., Moors, A. C., Matsick, J. L., y Valentine, B. (2013). A critical examination of popular assumptions about the benefits and outcomes of monogamous relationships. Personality and social psychology review : an official journal of the Society for Personality and Social Psychology, Inc, 17(2), 124-141. https://doi.org/10.1177/1088868312467087

Conley, T. D., Moors, A. C., Matsick, J. L., y Ziegler, A. (2012). The fewer the merrier?: Assessing stigma surrounding consensually non-monogamous romantic relationships. Analyses of Social Issues and Public Policy (ASAP), 13(1), 1-30. https://doi.org/10.1111/j.1530-2415.2012.01286.x

Cruz, M. (2014, junio 6). Club swinger en Costa Rica. Revista Perfil. https://www .revistaperfil.com/bienestar/amor-y-sexo/club-swinger-en-costa-rica-unaexperiencia-de-libertad/QHGUJM32ENCPHAJQ4HE5BS5KIQ/story/

Eisenberg, F. (1999). La infidelidad a lo largo del ciclo vital de la pareja. Publicación electrónica de la división de administración y ciencias sociales de la rectoría Zona Sur (ITESM), 1(2).

Flick, U., Von Kardorff, E. y Steinke, I. (2004) A Companion to Quialitative Research. SAGE Publications.

Gómez, J. (2009). Algunas cuestiones en torno a la subjetividad en el estilo de vida swinger. Apertura al debate. Revista Electrónica de Psicología Social "Poiésis", 18, 1-6. doi:ISSN 1692-0945

Gómez, J., y Martínez, C. (2009). La angustia masculina y la mujer tradicional en el estilo de vida Swinger: El ideal del amor romántico en época de la pornografía del goce. Ver en Subjetividad y estructura simbólica en el estilo de vida swinger. Universidad de San Buenaventura-Cali. http://borromeo.kennedy.edu.ar/Artculos/SwingersGallegoMart\%C3\%ADnez.pdf

Hurtado, C., y Serna, P. A. (2011). 5 El estilo de vida swinger y la noción de fidelidad: una lucha subjetiva entre libertad y exclusividad sexual. Textos \& Sentidos, (4). https://go.gale.com/ps/anonymous?id=GALE\%7CA334178596\&sid=googleScholar $\& v=2.1 \& i t=r \&$ linkaccess $=$ abs \&issn $=22158812 \& \mathrm{p}=\mathrm{IFME} \& \mathrm{sw}=\mathrm{w}$

Jamieson, L. (2004). Intimacy: Negotiated Non-Monogamy and the Limits of the Couple. En K. Duncombe, G. Harrison, y D. Marsden: The State of Affairs: Explorations in Infidelity and Commitment. Lawrence Erlbaum Associates, pp. 35-57.

Jenks, R. (2014). An Online Survey Comparing Swingers and Poliamorists. Electronic Hournal of Human Sexuality, 17. http://www .ejhs.org/volume17/swing.html.

Kimberly, C. y Hans, J. (2017). From Fantasy to Reality: a Grounded Theory of Experiences in Swinging Lifestyle. Archives of Sexual Behavior, 46(3), 789-799. doi:10.1007/s10508-015-0621-2.

Klesse, C. (2006). Polyamory and Its 'Others': Constesting the Terms of Non-Monogamy. Sexualities, 9(5), 565-583.

Lano, K. y Parry, C. (1995). Breaking the Barriers to Desire: New Approaches to Multiple Relationships. Five Leaves Publications.

Martínez, M. (1998). La investigación cualitativa etnográfica en educación. Trillas. 
Psicolnnova, 5(1), 2021, 27-45

Mastick, J., Conley, T., Ziegler, A., Moors, A. y Rubin, J. (2014). Love and Sex: Polyamorous Relationships are Perceived More Favourably than Swinging and Open

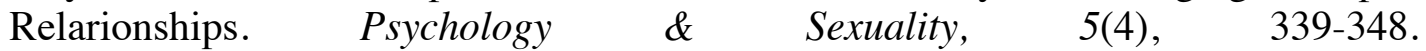
doi:http://dx.doi.org/10.1080/19419899.2013.832934

Noël, M. J. (2006). Progressive Polyamory: Considering Issues of Diversity. Sexualities, 9(5), 602-620. https://doi.org/10.1177/1363460706070003

Monsalve, A. (2009). Estilo de vida swinger. Universidad San Buenaventura Medellín.

Monsalve, A., Villa, A. y Álvarez, J. (2014). Algunos aspectos de estilo de vida swinger en una muestra de sujetos en la ciudad de medellín. Revista Virtual de Ciencia Sociales y Humanas "Psicoespacios", 8(12), 258-274. doi:ISSN 2145-2776

Olivera, P. (2014). La práctica swinger en la metrópolis. Universidad de la República de Uruguay. http://cienciassociales.edu.uy/wp-content/uploads/2014/09/Olivera.pdf

Orejuela, J., Piedrahita, J. y Renza, F. (2012). La práctica/estilo de vida swinger: ¿una práctica social-sexual perversa? Sexualidad, Salud y Sociedad - Revista Latinoamericana, 10, 37-69. doi:ISSN 1984-6487

Otey, J. (2017, agosto 15). Crucero para "parejas de mente abierta" navega el Mediterráneo. Crhoy.com. https://www.crhoy.com/entretenimiento/crucero-para-parejas-de-menteabierta-navega-el-mediterraneo/

Otey, J. (2018, mayo 30). Dueño de club "swinger" está desolado por incendio de local en San José. Crhoy.com. https://www.crhoy.com/nacionales/dueno-de-club-swingeresta-desolado-por-incendio-de-local-en-san-jose/

Otey, J. (2018, junio 10). Un swinger se confiesa: "Sexo para disfrutar, la regla es prohibido prohibir." Crhoy.com. https://www.crhoy.com/reportaje-especial/un-swinger-seconfiesa-sexo-para-disfrutar-la-regla-es-prohibido-prohibir

Pines, M. (1992). Romantic Jealousy: Five Perspectives and an Integrative Approach. Psychotherapy: Theory, Research, Practice, Training, 29(4), 675-683. doi:10.1037/0033-3204.29.4.675.

Vanegas, J. (2011). La dinámica vincular celos-infidelidad. Pensamiento Psicológico. 97102 